\title{
Mortalidad adolescente por causas violentas y Necesidades Básicas Insatisfechas. Argentina, trienios 2006-2008 y 2012-2014
}

\author{
Eleonora Soledad Rojas-Cabrera ${ }^{1-3}$ iD \\ ${ }^{1}$ Consejo Nacional de Investigaciones Científicas y Técnicas (CONICET), Buenos Aires, Argentina \\ ${ }^{2}$ Universidad Nacional de Córdoba (UNC), Córdoba, Argentina \\ ${ }^{3}$ Centro de Investigaciones y Estudios sobre Cultura y Sociedad (CIECS). Córdoba, Argentina
}

Cómo referenciar este artículo/

How to reference this article:
Rojas Cabrera E. Mortalidad adolescente por causas violentas y Necesidades Básicas Insatisfechas. Argentina, trienios 2006-2008 y 2012-2014. Mem. Inst. Investig. Cienc. Salud. 2021; 19(1): 64-73

\section{RES U ME N}

A partir de considerar el incremento de la violencia en el mundo, sus implicancias para la población joven y la escasez de estudios relativos al tema según sector socioeconómico, se planteó el objetivo de analizar la mortalidad adolescente por causas violentas según nivel de Necesidades Básicas Insatisfechas (NBI), en Argentina, entre los trienios 2006-2008 y 2012-2014. Para ello, se llevó a cabo un estudio cuantitativo y descriptivo, desde un enfoque sociodemográfico, con base en información estadística oficial. Se calcularon tasas de mortalidad para la población de 10 a 19 años, desagregadas por nivel de NBI, sexo, edad y grupo de causas violentas. Los resultados mostraron un incremento de la mortalidad para el conjunto de causas violentas independientemente del nivel de NBI, mayormente en varones de 15 a 19 años. En particular, aumentó la mortalidad por accidentes de transporte y eventos de intención no determinada (todos los niveles de NBI), otros accidentes y suicidios (mayores niveles de NBI) y homicidios (menores niveles de NBI). Surge así la necesidad de desplegar acciones "a medida" para reducir la mortalidad en todos los casos.

Palabras clave: Adolescencia, violencia, mortalidad, estatus socioeconómico, condiciones de vida, Argentina

\section{Adolescent mortality from violent causes and Unsatisfied Basic Needs. Argentina, 2006-2008 and 2012-2014 triennia}

\begin{abstract}
A B S T R A C T
Given the increase in violence in the world, its implications for the young population and the scarcity of studies related to this subject according to socioeconomic sector, the objective of this paper was to analyze adolescent mortality from violent causes by level of Unsatisfied Basic Needs (UBN) in Argentina, between triennia 2006-2008 and 2012-2014. For this purpose, a quantitative and descriptive study was carried out, using a socio-demographic approach, based on official statistical information. Mortality rates were calculated for the population aged 10 to 19 , disaggregated by level of UBN, sex, age and group of violent causes. The results showed an increase in mortality considering all violent causes regardless of the level of UBN, mostly in males aged 15 to 19 years. In particular, mortality from transport accidents and events of undetermined intention (all levels of UBN), other accidents and suicides (higher levels

Fecha de recepción: Febrero 2021. Fecha de aceptación: Marzo 2021

*Autor correspondiente: Eleonora Soledad Rojas Cabrera. Consejo Nacional de Investigaciones Científicas y Técnicas (CONICET), Buenos Aires, Argentina. Universidad Nacional de Córdoba (UNC). Centro de Investigaciones y Estudios sobre Cultura y Sociedad (CIECS). Córdoba, Argentina

Email: eleonorarojascabrera@gmail.com.
\end{abstract}


of UBN) and homicides (lower levels of UBN) increased. Therefore, "tailored-made" actions must be taken to reduce mortality in all cases.

Keywords: Adolescence, violence, mortality, socioeconomic status, living conditions, Argentina

\section{INTRODUCCIÓN}

Los hechos de violencia comprometen seriamente el ejercicio de los derechos humanos, produciendo consecuencias fatales en las personas que los padecen. De allí que su prevención y tratamiento constituya una de las principales preocupaciones a nivel global $^{(1)}$, sobre todo en las Américas, donde los niveles de mortalidad asociados son considerablemente más elevados en comparación con el resto del mundo(2). En esta región, se destaca la población adolescente (es decir, la comprendida por personas de entre 10 y 19 años) por estar afectada especialmente por la problemática, con una estructura de muertes liderada por homicidios, suicidios y accidentes de transporte ${ }^{(3)}$. Esta situación es corroborada también al interior de los países que la componen, incluso desde décadas pasadas ${ }^{(4-13)}$.

En este contexto, la mortalidad adolescente por causas violentas emerge como una cuestión prioritaria en América Latina y el Caribe. De allí que el Consenso de Montevideo sobre Población y Desarrollo, celebrado en 2013 en el ámbito de la Comisión Económica para América Latina y el Caribe ${ }^{(14)}$, y en consonancia con los lineamientos de la Agenda 2030 para el Desarrollo Sostenible ${ }^{(15)}$, inste a los países a desplegar acciones en favor de su reducción, atendiendo a la desigualdad económica y social vigente en cada uno de ellos.

Como integrante de la región, Argentina registra altas y crecientes tasas de mortalidad adolescente por causas violentas, principalmente en los últimos años. Así lo documentan diversos estudios llevados a cabo para el país en su conjunto y a nivel local ${ }^{(5-13)}$. Sin embargo, ninguno de estos estudios considera a la desagregación de las muertes por sector socioeconómico, información fundamental para identificar necesidades específicas según el caso y establecer prioridades para la acción.

A partir de lo anterior, se planteó una investigación con el objetivo de analizar el comportamiento de la mortalidad adolescente por causas de muerte violentas según sector socioeconómico en los trienios 2006-2008 y 2012-2014. Dado que la información oficial sobre mortalidad presenta limitaciones para dar cuenta acerca de este último (sintetizada en registros estadísticos de defunción con altos niveles de omisión de respuesta en preguntas sobre educación y ocupación, dos aspectos relacionados con la situación económica y social de la población), se realizó una combinación de esta información (desagregada, a su vez, por sexo, edad, causa y departamento de residencia) y datos obtenidos en los dos últimos censos de población relativos al indicador de Necesidades Básicas Insatisfechas (NBI).

\section{MATERIALES Y MÉTODOS}

Diseño: Estudio exploratorio y descriptivo realizado desde una perspectiva sociodemográfica, con apoyo de fuentes de datos secundarias.

Fuentes de datos: Se utilizaron las bases de datos de mortalidad provenientes de la Dirección de Estadística e Información de Salud de la Nación (DEIS) para los trienios 2006-2008 y 2012-2014; las bases usuarias de los censos poblacionales de 2001 y 2010; y las estimaciones de población provinciales y departamentales por sexo y edad del Instituto Nacional de Estadísticas y Censos (INDEC) para 2007 y 2013.

Población de interés: Población de 10 a 19 años de la República Argentina.

Período bajo estudio: La elección del período bajo estudio (es decir, el conformado por los trienios 2006-2008 y 2012-2014) obedeció a la disponibilidad de información al momento de llevarse a cabo la investigación. Asimismo, se contempló que esta información refiriese a períodos equidistantes del último censo.

\section{Variables:}

Causa de muerte violenta: Definida según los códigos establecidos en la Décima Clasificación Internacional de Enfermedades (CIE 10) ${ }^{(16)}$, seleccionados con base en trabajos anteriores sobre el tema ${ }^{(4,12)}$. Concretamente: Accidentes de transporte (V01- 
V99); Otros accidentes (W00-W99 y X00-X59); Homicidio (X85-Y09); Suicidio (X60X84); y Eventos de intención no determinada (Y10-Y34).

Quintil de NBI: Se considera que una persona presenta Necesidades Básicas Insatisfechas (NBI) cuando habita un hogar que presenta al menos una de las siguientes características: a) vivienda inconveniente: cuando el hogar reside en habitaciones de inquilinato, hotel o pensión, viviendas no destinadas a fines habitacionales, viviendas precarias y otro tipo de vivienda (salvo las viviendas tipo casa, departamento o rancho); b) carencias sanitarias: cuando el hogar no posee retrete; c) hacinamiento crítico: cuando en el hogar hay más de tres personas por cuarto; d) inasistencia escolar: cuando al hogar tiene al menos un niño en edad escolar ( 6 a 12 años) que no asiste a la escuela; e) capacidad de subsistencia: cuando el hogar tienen cuatro o más personas por miembro ocupado y, a su vez, un jefe que no ha completado el tercer grado de escolaridad primaria ${ }^{(17)}$.

La información contenida en los registros estadísticos de defunción (cuya sistematización da origen a las bases de datos de mortalidad) no posibilita calcular el indicador de NBI, debido a que no incorpora toda la información necesaria para ello. De allí que este indicador, aplicado a las personas fallecidas, fuera obtenido con apoyo de los resultados del Censo de Población 2010, mediante la vinculación entre estos últimos y las bases de datos de mortalidad empleadas a partir de la variable departamento de residencia ${ }^{1}$, presente en ambas fuentes de datos. En otras palabras, se buscó aproximar el nivel de NBI de las personas fallecidas desde la proporción de la población con NBI al interior de cada unidad departamental según el censo en cuestión. Este procedimiento fue ejecutado siguiendo una investigación de Buchbinder (18) y permitió finalmente obtener la variable Quintil de NBI utilizada en este trabajo, con cinco (5) categorías, organizadas de menor a mayor proporción de NBI (es decir, de Quintil 1 a Quintil 5, en ese orden).

Sexo: Ligada a dos posibles categorías: Varones y Mujeres.

Grupo de edad: Con las categorías 10 a 14 años y 15 a 19 años, correspondientes, respectivamente, con las fases temprana y tardía de la adolescencia ${ }^{(3)}$.

Procedimientos: Se calcularon tasas específicas de mortalidad (TM) según las variables especificadas. Estas TM resultaron del cociente entre el promedio anual de las defunciones contabilizadas en cada trienio detallado y la población total asociada al año central de dicho trienio. En todos los casos, se expresaron por 100.000 habitantes, conforme a la unidad de medida empleada en estudios del tipo ${ }^{(19)}$.

Dado que el INDEC no publica estimaciones de población departamentales por edad, para obtener la población necesaria para el cálculos de las TM, se aplicaron los siguientes procedimientos: a) para 2007, se interpolaron linealmente los resultados de los censos de 2001 y 2010; y b) para 2013, se ajustó la distribución de la población por sexo y edad de cada departamento relevada en 2010 a las estimaciones provinciales y departamentales disponibles para ese año.

Todos los procedimientos fueron realizados con Microsoft Office ${ }^{\circledR}$, Excel $^{\circledR} 2007$ e IBM SPSS Statistics 23.0 (EE.UU.). Así también, con apoyo de las planillas AGEINT y CTBL 32 del paquete informático PAS desarrollado por la Oficina del Censo de EE.UU. (en inglés, U.S. Census Bureau) para el análisis de la población ${ }^{(20)}$.

\section{RESULTADOS}

La Tabla 1 contiene, para el total del país y por quintil de NBI, el total de defunciones registradas en la población adolescente en los trienios 2006-2008 y 20122014, el total atribuido a causas violentas y la población asociada en el año central contenido en cada trienio. Sobre la base de estos valores, se estructuran los indicadores que se presentan posteriormente.

\footnotetext{
${ }^{1}$ Político-administrativamente, la República Argentina se divide en 23 provincias y un (1) distrito federal (Ciudad Autónoma de Buenos Aires). A su vez, estas jurisdicciones se subdividen en 379 departamentos, 135 partidos y 15 comunas. A los fines de este trabajo, estas tres últimas categorías (528 en total) se consideran departamentos.
} 
Tabla 1: Defunciones adolescentes por todas las causas y por causas violentas, trienios 2006-2008 y 2012-2014. Población adolescente estimada al 1 de julio de 2007 y 2013. Argentina.

\begin{tabular}{|c|c|c|c|c|c|c|}
\hline \multirow[b]{2}{*}{ Indicador } & \multirow[b]{2}{*}{ Total país } & \multicolumn{3}{|c|}{ Quintil de NBI } & \multirow[b]{2}{*}{$\begin{array}{l}\text { Quintil } \\
\mathbf{4}\end{array}$} & \multirow[b]{2}{*}{ Quintil 5} \\
\hline & & Quintil 1 & Quintil 2 & Quintil 3 & & \\
\hline $\begin{array}{l}\text { Total defunciones, } \\
2006-2008\end{array}$ & 10.772 & 1.321 & 3.449 & 3.405 & 1.538 & 1.059 \\
\hline $\begin{array}{l}\text { Defunciones por } \\
\text { causas violentas, } \\
2006-2008\end{array}$ & 6.201 & 790 & 1.990 & 1.966 & 899 & 556 \\
\hline Población, 2007 & 6.885 .668 & 934.489 & 2.276 .130 & 2.241 .615 & 889.110 & 544.324 \\
\hline $\begin{array}{l}\text { Total defunciones, } \\
2012-2014\end{array}$ & 11.428 & 1.328 & 3.539 & 3.676 & 1.690 & 1.195 \\
\hline $\begin{array}{l}\text { Defunciones por } \\
\text { causas violentas, } \\
2012-2014\end{array}$ & 6.823 & 768 & 2.149 & 2.184 & 1.028 & 694 \\
\hline Población, 2013 & 7.081 .447 & 894.050 & 2.337 .144 & 2.341 .736 & 933.922 & 574.595 \\
\hline
\end{tabular}

Fuente: Elaboración propia con base en información provenientes de la DEIS y el INDEC.

La TM adolescente por causas violentas creció a mayor quintil de NBI. Es así que, en el trienio 2006-2008, las TM de los quintiles 1 a 3 se acercaron al valor promedio nacional, mientras que las restantes TM lo superaron. En el trienio 2012-2014, la TM se incrementó en todos los casos, siendo este incremento más pronunciado a mayor nivel de NBI (Tabla 2). En efecto, la TM creció 2\%, 5\%, 6\%, $9 \%$ y $18 \%$, respectivamente, considerando los quintiles de NBI de menor a mayor.

Tabla 2: Tasa de mortalidad (TM) adolescente por causas violentas según quintil de NBI (por 100.000 habitantes). Trienios 2006-2008 y 2012-2014, Argentina.

\begin{tabular}{lllllll}
\hline \multirow{2}{*}{ Período } & \multirow{2}{*}{ Total país } & \multicolumn{2}{l}{ Quintil de NBI } & & & \\
\cline { 2 - 7 } & & Quintil 1 & Quintil 2 & Quintil 3 & Quintil 4 & Quintil 5 \\
\hline $2006-2008$ & 30 & 28,2 & 29,1 & 29,2 & 33,7 & 34 \\
$2012-2014$ & 32,1 & 28,6 & 30,6 & 31,1 & 36,7 & 40,2 \\
\hline
\end{tabular}

Fuente: Elaboración propia con base en información provenientes de la DEIS y el INDEC.

Al incorporar el sexo y la edad de la población adolescente fallecida, se registraron TM superiores en la población de 15-19 años, principalmente en la masculina. Este patrón se observó con independencia del quintil de NBI, aunque con mayores TM a mayor quintil de NBI (Tabla 3).

Las diferencias en detrimento de varones de 15-19 años tendieron a agudizarse, al aumentar la TM asociada en todos los casos (nuevamente, con más énfasis en los quintiles de mayor NBI; un $12 \%, 16 \%, 13 \%, 24 \%$ y $31 \%$, respectivamente); y descender en la población de 10-14 años de ambos sexos. Por su parte, la TM femenina de 15-19 años se comportó disímilmente: decreció en los quintiles de NBI 1 , 2 y 4 ; y aumentó en los quintiles 3 y 5 , especialmente en este último (Tabla 3 ). 
Tabla 3: Tasa de mortalidad (TM) adolescente por causas violentas según quintil de NBI, sexo y grupo de edad (por 100.000 habitantes). Trienios 2006-2008 y 20122014. Argentina.

\begin{tabular}{|c|c|c|c|c|c|c|c|c|}
\hline \multirow{2}{*}{ Sexo } & \multirow{2}{*}{$\begin{array}{l}\text { Grupo } \\
\text { de edad }\end{array}$} & \multirow{2}{*}{ Período } & \multirow{2}{*}{$\begin{array}{l}\text { Total } \\
\text { país }\end{array}$} & \multicolumn{5}{|c|}{ Quintil de NBI } \\
\hline & & & & Quintil 1 & Quintil 2 & Quintil 3 & Quintil 4 & Quintil 5 \\
\hline \multirow{4}{*}{$\begin{array}{l}\text { y } \\
\stackrel{5}{0} \\
\frac{1}{0} \\
>\end{array}$} & \multirow{2}{*}{$\begin{array}{l}10-14 \\
\text { años }\end{array}$} & $2006-2008$ & 17 & 16,1 & 15,9 & 15,3 & 21,3 & 22,6 \\
\hline & & $2012-2014$ & 13,1 & 11 & 12,6 & 12,3 & 15 & 18,6 \\
\hline & \multirow{2}{*}{$\begin{array}{l}15-19 \\
\text { años }\end{array}$} & $2006-2008$ & 73,6 & 70,7 & 73,6 & 72,3 & 77,2 & 78,7 \\
\hline & & $2012-2014$ & 86,1 & 79,1 & 85,2 & 81,9 & 95,6 & 103,3 \\
\hline \multirow{4}{*}{ 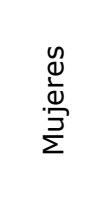 } & \multirow{2}{*}{$\begin{array}{l}10-14 \\
\text { años }\end{array}$} & $2006-2008$ & 9 & 7,4 & 8,2 & 8,4 & 10,6 & 15 \\
\hline & & $2012-2014$ & 7,1 & 5,5 & 6,2 & 6,7 & 10 & 9,8 \\
\hline & \multirow{2}{*}{$\begin{array}{l}15-19 \\
\text { años }\end{array}$} & $2006-2008$ & 20,4 & 17,3 & 17,9 & 21,2 & 27,4 & 22,8 \\
\hline & & $2012-2014$ & 20,4 & 16,8 & 16,4 & 21,9 & 25 & 29,9 \\
\hline
\end{tabular}

Fuente: Elaboración propia con base en información proveniente de la DEIS y el INDEC.

Un análisis de las muertes por causas reveló nuevas coincidencias y disparidades según quintil de NBI. Por una parte, predominaron las muertes por Accidentes de tránsito, con TM crecientes en la mayoría de los casos (Figura 1; Tabla 4).

Seguidamente, aparecieron las muertes por Otros accidentes. Sin embargo, a diferencia de la primera, esta causa se vinculó a TM decrecientes en casi todos los quintiles de NBI, hecho que incidió en el descenso de su participación relativa en el total de muertes violentas. Excepcionalmente, en el Quintil 5, la TM aumentó (Figura 1; Tabla 4).

Continuaron, en orden de relevancia, los Suicidios, con TM y peso relativo crecientes, fundamentalmente debido al aumento de la TM específica en los quintiles de NBI 3 a 5 . En otro extremo, las TM específicas de dos primeros quintiles de NBI se redujeron, así como también su participación relativa en el total de muertes estudiadas (Figura 1; Tabla 4).

Por último, aunque las TM por Homicidios y Eventos de intención no determinada se relacionaron, en general, a valores más bajos, llamaron la atención por su crecimiento en los primeros tres y cuatro quintiles de NBI, respectivamente. Este incremento, se tradujo, a su vez, en un aumento de la participación de estas muertes en cada caso (Figura 1; Tabla 4).

Fuente: Elaboración propia con base en información proveniente de la DEIS y el INDEC.

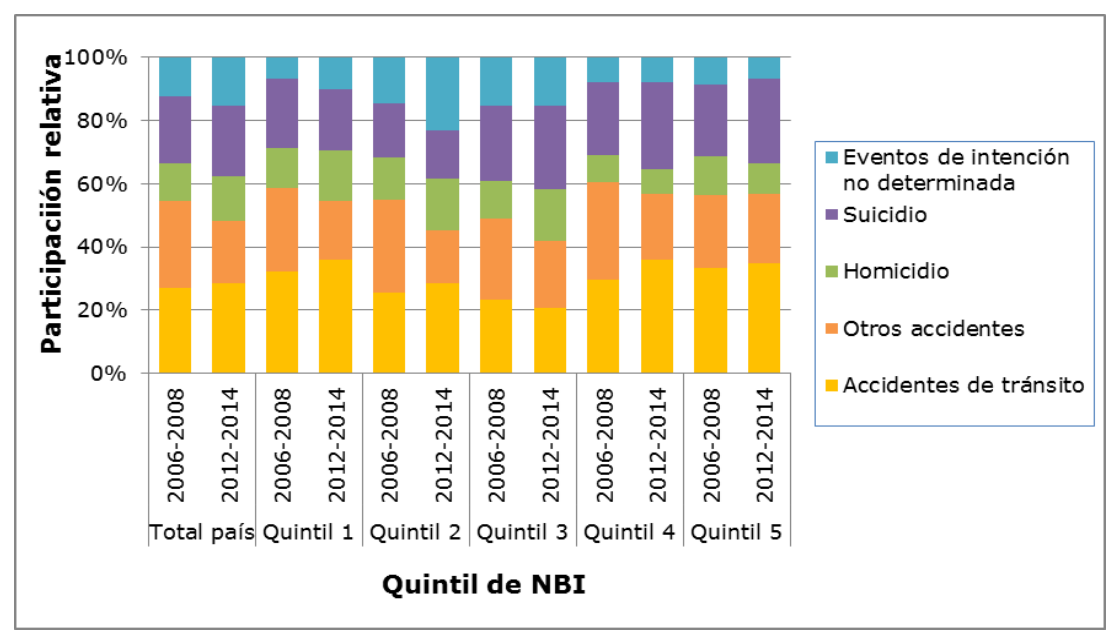

Figura 1: Distribución de las muertes adolescentes por causas violentas según quintil de NBI y causas seleccionadas (\%). Trienios 2006-2008 y 2012-2014. Argentina. 
Tabla 4: Argentina. TM adolescente por causas violentas (por 100.000 habitantes) según quintil de NBI y causas seleccionadas. Trienios 2006-2008 y 2012-2014

\begin{tabular}{lccccccc}
\hline \multirow{2}{*}{ Causa } & \multirow{2}{*}{ Período } & Total & \multicolumn{5}{c}{ Quintil de NBI } \\
\cline { 5 - 8 } & & país & $\begin{array}{c}\text { Quintil } \\
\mathbf{1}\end{array}$ & $\begin{array}{c}\text { Quintil } \\
\mathbf{2}\end{array}$ & $\begin{array}{c}\text { Quintil } \\
\mathbf{3}\end{array}$ & $\begin{array}{c}\text { Quintil } \\
\mathbf{4}\end{array}$ & $\begin{array}{c}\text { Quintil } \\
\mathbf{5}\end{array}$ \\
\hline Accidentes de & $2006-2008$ & 8,1 & 9,1 & 7,5 & 6,8 & 9,9 & 11,4 \\
transporte & $2012-2014$ & 9,2 & 10,3 & 8,8 & 6,4 & 13,2 & 14,1 \\
Otros accidentes & $2006-2008$ & 8,3 & 7,5 & 8,6 & 7,5 & 10,4 & 7,8 \\
& $2012-2014$ & 6,3 & 5,4 & 5,1 & 6,7 & 7,7 & 8,8 \\
Homicidio & $2006-2008$ & 3,6 & 3,5 & 3,8 & 3,5 & 2,9 & 4,2 \\
Suicidio & $2012-2014$ & 4,6 & 4,5 & 5 & 5 & 2,9 & 3,8 \\
Eventos de & $2006-2008$ & 6,4 & 6,2 & 5 & 6,9 & 7,8 & 7,7 \\
intención no & $2012-2014$ & 7,2 & 5,5 & 4,6 & 8,2 & 10,1 & 10,8 \\
determinada & $2006-2008$ & 3,7 & 1,9 & 4,3 & 4,5 & 2,7 & 3,0 \\
\hline
\end{tabular}

Fuente: Elaboración propia con base en información proveniente de la DEIS y el INDEC.

\section{DISCUSIÓN}

Los resultados obtenidos son consistentes con estudios previos que dan cuenta, para Argentina en su conjunto, acerca de un incremento de la mortalidad adolescente por causas violentas en general, principalmente en varones y con más énfasis en el grupo de 15-19 años ${ }^{(5,7-12)}$. Asimismo, con otros trabajos que señalan que las poblaciones con mayor proporción de NBI presentan mayor el riesgo de morir por este tipo de causas ${ }^{(18,21-25)}$. Por el contrario, difieren de otras investigaciones que indican que la relación entre el nivel de NBI y la mortalidad en cuestión es negativa ${ }^{(26-28)}$.

Respecto de las razones que motivarían el comportamiento de la mortalidad adolescente por accidentes de transporte, de trayectoria indefinida según el quintil de NBI, deberían contemplarse diversos factores. Entre ellos, el modo de vinculación de las personas con el tránsito según su nivel socioeconómico, no solo desde el medio de transporte más utilizado, sino también desde el (in)cumplimiento de las normas de seguridad vial y las condiciones en que este se produce ${ }^{(29-31)}$.

La utilización diferencial de medios de transporte se evidencia, por ejemplo, en el mayor empleo de motocicletas por parte de los sectores más pobres (incluso, más acentuado en los últimos años, dadas las facilidades que se han concedido para la adquisición de estos vehículos en el país ${ }^{(30-31)}$. Teniendo en cuenta, a su vez, que se corresponde con un medio de transporte de uso más extendido en jóvenes, podría afirmarse que el riesgo de sufrir accidentes por esta vía (y morir en consecuencia) sería más elevado en esta población, sobre todo a menor nivel socioeconómico ${ }^{(30-31)}$.

Respecto del (in)cumplimiento de normas de seguridad vial, merece destacarse la falta de importancia que suele asignarse al uso del cinturón de seguridad en personas menores de edad ${ }^{(30)}$. Ello podría influir en el incremento del nivel de muertes adolescentes por accidentes en la mayoría de los quintiles de NBI, por lo que debería ser especialmente considerado para disminuir su valor.

Adicionalmente, el consumo de alcohol en adolescentes al volante imprime sus consecuencias. En efecto, se trata de una población que generalmente no percibe el riesgo, que suele adoptar la cultura del alcohol como medio de sociabilidad y que, usualmente, no es apropiadamente controlada ni sancionada por el Estado, cuando corresponde ${ }^{(30)}$.

En otro orden, los resultados consignados, a diferencia de algunos estudios previos $^{(32)}$, revelan que la mortalidad por homicidios es superior en los primeros quintiles de NBI, donde son mayores las posibilidades económicas y es menor la presencia del Estado como organismo de control ${ }^{(28,33)}$. Esta realidad se potenciaría al considerar, además, la presencia de sectores de la población carentes de políticas 
sociales y con marcada segregación entre "ricos" y "pobres", donde el delito configura un medio de acceso al consumo y al reconocimiento social ${ }^{(33-34)}$, y en los cuales subyacen otros factores estructurales de inequidades en salud como el abandono escolar y la falta de trabajo ${ }^{(25)}$.

La influencia de estos dos últimos factores impactaría, a su vez, en la mayor mortalidad por suicidios que registran los quintiles de mayor proporción de NBI, aunados a la vivencia de situaciones traumáticas (inestabilidad familiar y discriminación, entre otras) que derivan en la falta de autoestima personal, la ausencia de oportunidades en general (35-38) y una mayor propensión a desplegar conductas de riesgo como el consumo de sustancias problemáticas ${ }^{(40)}$. No menos importante resulta la inexistencia de una política pública integral para el abordaje del suicidio adolescente en el país, a nivel nacional y subnacional ${ }^{(40)}$.

Todas estas lecturas deben hacerse considerando, simultáneamente, las falencias del sistema estadístico oficial de Argentina para dar cuenta, cabalmente, de las muertes por causas violentas. Estas falencias se efectivizan, por ejemplo, en el volumen de muertes asignadas a la categoría "Eventos de intención no determinada", que no posibilita separar las muertes accidentales de las intencionales ${ }^{(39-42)}$, y que no consigue reducir su valor aunque experimenta mejorías para algunos años ${ }^{(40)}$. Además, en los altos niveles de muertes atribuidas a "causas mal definidas" y "poco útiles", que dificultan igualmente la toma de decisiones informadas y acertadas para contrarrestar la violencia en tanto causa de muerte y problema de salud pública ${ }^{(42-44)}$.

Más allá de las limitaciones de los datos, los diferenciales en el nivel, la dinámica y la composición de las muertes violentas según quintil de NBI aquí documentados ameritan el despliegue de acciones "a medida" con énfasis en los factores que más intervienen en su ocurrencia. Ello exige contar no solo con un registro de defunciones fortalecido en su calidad sino también con relevamientos especializados que permitan vincular, en profundidad, la ocurrencia de las muertes con las características de cada contexto en particular, a fin de orientar los recursos disponibles con acuerdo de las necesidades específicas según el caso.

\section{REFERENCIAS BIBLIOGRÁFICAS}

1. Organización Panamericana de la Salud / Organización Mundial de la Salud. Informe mundial sobre la violencia y la salud: resumen. Washington, D.C.: Organización Mundial de la Salud; 2002.

2. United Nations Office on Drugs and Crime. Global Study on Homicide 2019. Homicide trends, patterns and criminal justice response. Vienna: United Nations Office on Drugs and Crime; 2019.

3. Organización Panamericana de la Salud. La salud de los adolescentes y jóvenes en la Región de las Américas: la aplicación de la estrategia y el plan de acción regionales sobre la salud de los adolescentes y jóvenes (20102018).Washington, D.C.: Organización Panamericana de la Salud; 2018.

4. Yunes J, Zubarew, T. Mortalidad por causas violentas en Adolescentes y Jóvenes: Un Desafío para la Región de las Américas. Rev. bras. epidemiol. [Internet] 1999 diciembre. [acceso 17 de octubre de 2020]; 2 (3): 102-171. Disponible en: 10.1590/S1415790X1999000200002

5. Serfaty E, Foglia V, Masaútis A, Negri G. Mortalidad por causas violentas en adolescentes y jóvenes de Argentina, 1991-2000. Vertex. 2003; 14(2): 4048.

6. Freitez A, Romero D. Comparación de dos países con mayor crecimiento de la mortalidad juvenil por causas violentas: Brasil y Venezuela. Trabajo presentado en I Congreso de la Asociación Latinoamericana de Población; 2004 Septiembre 18-20; Caxambú, Brasil. [Internet] [acceso 18 de marzo de 2010]. Disponible en: http://www.alapop.org/alap/images/P DF/ALAP2004 361.pdf

7. Burrone $M$, Bella $M$, Acosta $L$, Villace B, López de Neira M, Fernández R, Enders J. Estudio de muertes por causas violentas: un análisis de la tendencia en jóvenes, Argentina, 2000-2008. Cad. saúde colet. [Internet] 2012. [acceso 11 de octubre de 2020]; 20(4), 460-465. Disponible en: $10.1590 / \mathrm{S} 1414-$ 462X2012000400009

8. Bella M, Acosta L, Villace B, López de Neira $M$, Enders J, Fernández R. Análisis de la mortalidad por suicidio en niños, adolescentes y jóvenes. Argentina, 2005-2007. Arch Argent Pediatr. [Internet] 2013. [acceso 26 
de septiembre de 2020]; 111(1), 1621. Disponible en: 10.5546/aap.2013.16

9. Cardona D, Peláez E, Aidar T, Ribotta B, Alvarez M. Mortalidad por causas externas en tres ciudades latinoamericanas: Córdoba (Argentina), Campinas (Brasil) y Medellín (Colombia), 1980-2005. Rev. bras. estud. popul. [Internet] juliodiciembre 2008. [acceso 17 de septiembre de 2020]; 25(2): 335-352. Disponible en: 10.1590/S010230982008000200009

10. Cardona-Arango D, Escanés G, Fantín M, Peláez E. Mortalidad por causas externas: un problema de salud pública. Argentina, Chile y Colombia. 2000-2008. Poblac. Salud Mesoam. [Internet] enero-junio 2013. [acceso 12 de septiembre de 2020]; 10(2): informe técnico 2. Disponible en: https://revistas.ucr.ac.cr/index.php/ps m/article/view/8518/8043

11. Pantelides E, Govea J, Gaudio M, Bruno M. Mortalidad adolescente por homicidio. Argentina y provincia de Buenos Aires. Trabajo presentado en VI Congreso de la Asociación Latinoamericana de Población; 2014 Agosto 12-15; Lima, Perú. [Internet] [acceso 19 de julio de 2018]. Disponible en: http://www.alapop.org/Congreso2014 LDOCSFINAIS PDF/ALAP 2014 FINAL 97.pdf

12. Rojas Cabrera E. Mortalidad por causas violentas en adolescentes y jóvenes de dos ciudades del Cono Sur: Córdoba (Argentina) y Porto Alegre (Brasil), 1990-2010. [Internet] enero 2015. [acceso 10 de noviembre de 2019]; 20(1), 29-37. Disponible en: $10.1590 / 1413-$

81232014201.19162013

13. Fondo de las Naciones Unidas para la Infancia. Situación en la Argentina. Buenos Aires: Fondo de las Naciones Unidas para la Infancia; 2019.

14. Comisión Económica para América Latina y el Caribe. Consenso de Montevideo sobre población y desarrollo. Montevideo: Naciones Unidas; 2013.

15. Naciones Unidas. Resolución aprobada por la Asamblea General el 25 de septiembre de 2015. Transformar nuestro mundo: la Agenda 2030 para el Desarrollo Sostenible. A/RES/70/1. Nueva York: Naciones Unidas; 2015.

16. Organización Panamericana de la Salud / Organización Mundial de la Salud. Clasificación Estadística internacional de enfermedades y problemas relacionados con la salud. Décima revisión. Washington, D.C.: Organización Mundial de la Salud; 1995.

17. Dirección Nacional de Relaciones Económicas con las Provincias Ministerio de Economía y Finanzas Públicas de la Nación, Argentina. Necesidades Básicas Insatisfechas (NBI). Información censal del año 2010 (Versión ampliada con datos departamentales). Buenos Aires: Ministerio de Economía y Finanzas Públicas de la Nación; 2014.

18. Buchbinder M. Mortalidad infantil y desigualdad socioeconómica en la Argentina. Tendencia temporal. Arch Argent Pediatr. [Internet] 2008 junio. [acceso 9 de julio de 2020]; 106(3), 212-218. Disponible en: https://www.sap.org.ar/docs/publicaci ones/archivosarg/2008/v106n3a05.pd f

19. Organización Panamericana de la Salud - Unidad de Información y Análisis de Salud. Iniciativa Regional de Datos Básicos en Salud; Glosario de Indicadores. Washington D.C.: Organización Panamericana de la Salud; 2015.

20. Arriaga E. El análisis de la población con microcomputadoras. Córdoba, Argentina: Universidad Nacional de Córdoba; 2001.

21. Campo-Arias A, Herazo E. Pobreza, desigualdad y tasa de suicidio en Colombia, 2012. Duazary. [Internet] 2014 julio-diciembre. [acceso 5 de enero 2021]; 11(2): 126-130. Disponible en: https://revistas.unimagdalena.edu.co/ index.php/duazary/article/view/819/7 $\underline{37}$

22. Page A, Morrell S, Taylor R. Suicide differentials in Australian males and females by various measures of socioeconomic status, 1994-98. Aust N Z J Public Health. [Internet] 2002 August. [acceso 6 de enero 2021]; 26(4):318$324 . \quad$ Disponible en: https://onlinelibrary.wiley.com/doi/ep df/10.1111/j.1467842X.2002.tb00178.x

23. Inoue $K$, Fujita $Y$, Takeshita $H$, Abe $S$, Fujihara J, Ezoe S, et al. A Long-term Study of the Association between the Relative Poverty Rate and Suicide Rate in Japan. J Forensic Sci. [Internet] 2016. [acceso 6 de enero 2021]; 61(S1): S140-S143. Disponible en: 10.1111/1556-4029.12998

24. Blandon-Paternina, M. Relación existente entre la pobreza y la violencia en Cartagena de Indias, 
período 2000-2006. [monografía en Internet]. Barranquilla: Universidad del Norte. Programa de Maestría en Desarrollo Social; 2009 [acceso 27 de diciembre de 2020]. Disponible en: https://manglar.uninorte.edu.co/handl e/10584/668

25. Oyola-García A, Berto-Gonzáles M, Valdez-Huarcaya W. La muerte por violencia en El Huallaga: un posible efecto de los determinantes estructurales de las inequidades en salud. An. Fac. med. [Internet] 2016 octubre-diciembre [acceso 11 de enero 2021]; 77(4): 423-424. Disponible en: http://www.scielo.org.pe/pdf/afm/v77 n4/a18v77n4.pdf

26. Dávila-Cervantes CA, Pardo-Montaño AM. Impacto de factores socioeconómicos en la mortalidad por suicidios en Colombia, 2000-2013. Rev Gerenc Polít Salud. [Internet] 2017. [acceso 4 de enero 2021]; 16(33): 36-51. Disponible en: 10.11144/Javeriana.rgps16-33.ifsm

27. Quinlan-Davidson $M$, Sanhueza $A$, Espinosa I, Escamilla-Cejudo JA, Maddaleno $M$. Suicide among young people in the Americas. J Adolesc Health. [Internet] 2014. [acceso 14 de enero 2021]; 54(3): 262-268. Disponible en: 10.1016/j.jadohealth.2013.07.012

28. Joiner TE. Why people die by suicide. Cambridge: Harvard University Press; 2005.

29. Macías G, Almedia-Filho N, Alazraqui M. Análisis de las muertes por accidentes de tránsito en el municipio de Lanús, Argentina, 1998-2004. Salud colectiva. [Internet] 2010 septiembre-diciembre. [acceso $10 \mathrm{de}$ enero de 2021]; 6(3): 313-328. Disponible en: 10.18294/sc.2010.287

30. Ministerio de Transporte - Presidencia de la Nación. Situación de la seguridad vial en Argentina. Datos y análisis para un abordaje integral del problema. Información disponible hasta el año 2016. Buenos Aires: Ministerio de Transporte - Presidencia de la Nación; 2018.

31. Andrada $\mathrm{MJ}$, Bertone $\mathrm{CL}$. Análisis de las defunciones por accidentes de tránsito en los registros oficiales de La Rioja - Argentina. Periodo 2011 2013. Mem. Inst. Investig. Cienc. Salud. [Internet] 2017. [acceso $10 \mathrm{de}$ octubre de 2020]; 15(2): 6-13. Disponible en: http://archivo.bc.una.py/index.php/RI IC/article/view/1223/1121
32. Spinelli $H$, Alazraqui M, Macías G, Zunino G, Nadalich J. Muertes violentas en la Ciudad de Buenos Aires. Una mirada desde el Sector Salud. Centro de Estudios de Estado y Sociedad. Seminario VI. Serie Seminarios Salud y Política Pública. Buenos Aires: Centro de Estudios de Estado y Sociedad; 2005.

33. Sperberg J, Happe B. Violencia y delincuencia en barrios pobres de Santiago de Chile y Río de Janeiro. Nueva sociedad. [Internet] 2000 septiembre-octubre. [acceso 5 de enero de 2021]; 169: 44-60. Disponible en: https://nuso.org/articulo/violencia-ydelincuencia-en-barrios-pobres-desantiago-de-chile-y-rio-de-janeiro/

34. Pegoraro JS. Notas sobre los jóvenes portadores de la violencia juvenil en el marco de las sociedades posindustriales. Sociologias. [Internet] 2002 diciembre. [acceso 7 de enero de 2021]; 8: 276-317. Disponible en: 10.1590/S1517-45222002000200012

35. Meneghel SN, Victora CS, Faria NMX, Carvalho LA, Falk JW Características epidemiológicas do suicídio no Rio Grande do Sul. Rev. Saúde Pública [Internet]. 2004 diciembre. [acceso 4 de enero de 2021]; 38(6): 804-810. Disponible en: 10.1590/S003489102004000600008

36. Stack S. Suicide: A decade review of the sociological literature. Deviant Behavior. 1982; 4(1):41-66.

37. Ortega PA, Manrique RD, Tovilla CA, López C, Cuartas JM. Clinical and epidemiological characteristics of suicides committed in Medellin, Colombia. rev.colomb.psiquiatr. [Internet]. 2014 abril. [acceso 28 de diciembre de 2020]; 43(2):106-112. Disponible en: http://www.scielo.org.co/scielo.php?sc ript $=$ sci arttext\&pid $=$ S003474502014000200007

38. World Health Organization. Public health action for the prevention of suicide. A framework. Ginebra: World Health Organization; 2012.

39. Santillán-Pizarro MM, Pereyra EJ. Factores asociados a la ideación del suicidio en adolescentes. Algunas evidencias en Argentina. Astrolabio [Internet]. 2021 enero. [acceso 7 de enero de 2021]; 26: 160-181. Disponible en: https://revistas.unc.edu.ar/index.php/ astrolabio/article/view/27659

40. Alazraqui $M$, Spinelli $H$, Zunino MG, Souza ER. Calidad de los sistemas de información de mortalidad por 
violencias en Argentina y Brasil 1990-2010. Ciênc. saúde coletiva [Internet]. 2012 diciembre. [acceso 7 de enero de 2021]; 17(2): 32793288. Disponible en: $10.1590 / \mathrm{S} 1413-$ 81232012001200013

41. Altieri D. Mortalidad por suicidios en Argentina Nivel, tendencia y diferenciales. Trabajo presentado en IX Jornadas Argentinas de Estudios de Población; 2007 Noviembre 1-2; Huerta Grande, Córdoba, Argentina. [Internet] [acceso 25 de noviembre de 2020]. Disponible en: http://www.redaepa.org.ar/jornadas/i xjornadas/resumenes/Se06-Mortalidad_Fantin/mesa-6b/Altieri.pdf

42. Santoro A. Recálculo de las tendencias de mortalidad por accidentes, suicidios y homicidios en Argentina, 19972018. Rev Panam Salud Pública [Internet]. 2020. [acceso 8 de enero de 2021]; 44:e74. Disponible en: 10.26633/RPSP.2020.74
43. Dirección de Estadísticas e Información de Salud - Ministerio de Salud de la Nación Argentina [sede Web]. Buenos Aires: Dirección de Estadísticas e Información de Salud; 2021 [actualizado 15 de enero de 2021; acceso 15 de enero de 2021]. Reporte Interactivo de Estadísticas de Salud. Disponible en: http://deis.msal.gov.ar/ReporteIntera ctivo/

44. Ribotta BS. Causas de defunción mal definidas en las provincias de Argentina, 2001-2013. Mem. Inst. Investig. Cienc. Salud. [Internet] 2016. [acceso 8 de agosto de 2020]; 14(3): 86-95. Disponible en: http://archivo.bc.una.py/index.php/RI IC/article/view/1110/961 